\title{
Stress-strain state of spherical shells with two unequal holes
}

\author{
Nurilla Noraliev ${ }^{1, *}$, Bakhrom Ishniyazov ${ }^{1}$, Bunyod Safarov $^{1}$, and Oybek Saparov ${ }^{1}$ \\ ${ }^{1}$ Tashkent State Agrarian University, University str., 2, Tashkent province, Uzbekistan, 100140
}

\begin{abstract}
This article presents the results of a numerical study of the stress concentration around two equal and unequal holes in an orthotropic spherical shell made of composite materials under the action of internal pressure. The influence of geometric (hole radii, shell thickness, distance between holes) characteristics, as well as material orthotropy and shear stiffness, on the stress state of spherical shells made of composite materials is studied. A numerical algorithm based on the finite element method has been developed and a software package has been implemented on a computer that allows solving the problem of stress concentration near two unequal holes in spherical shells made of composite materials.
\end{abstract}

\section{Introduction}

A fairly large number of works are devoted to the methods of calculating shell structures made of composite materials and the study of stress concentration near holes. However, most of the studies were carried out within the framework of the classical Kirchhoff-Love hypothesis, which does not take into account interlayer and transverse shears characteristic of composite materials $[1,2]$.

Within the framework of the improved theory of the Timoshenko type, the application of the finite element method is proposed for solving problems of orthotropic shells made of composite materials, weakened by several holes [3,4].

Multiply connected shells made of composite materials have been considered by a few authors. The main results are given in [2, 7-9]. For an orthotropic cylindrical shell with several holes, a solution was constructed in the work of K I Shnerenko, N Kh Noraliev [1014]. Moreover, the distribution of stresses around two equal circular holes in an orthotropic spherical shell made of a composite material was investigated taking into account shear stiffness [5, 6]. As follows from the above review of works, at present, the study of the stress-strain state of shells made of composite materials with two unequal holes is insufficient.

Therefore, in this paper, we study the stress distribution around two unequal circular holes in an orthotropic spherical shell made of a composite material. Using the refined theory of Timoshenko-type shells, we will take into account the effect of transverse shear deformations for the entire package of the shell as a whole. In this case, the composite material will be considered as homogeneous with the given characteristics.

\footnotetext{
*Corresponding author: n.x.noraliev@yandex.com
} 


\section{Materials and methods}

A spherical shell with two unequal circular holes of radii $r_{01}$ and $r_{02}$ (Fig. 1) and intensity $p_{0}$ under the influence of internal pressure e is considered. The study area is divided into finite elements using the finite element method $[5,6]$.

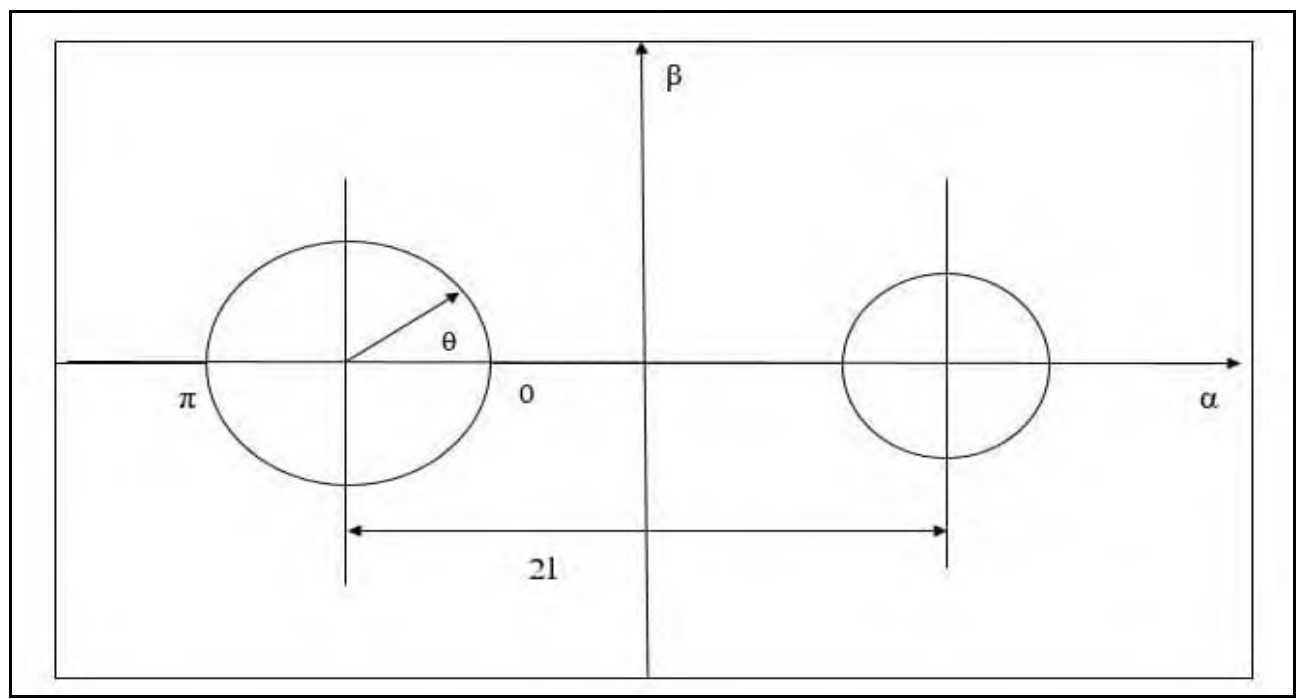

Fig. 1. Spherical shell with two unequal circular holes.

In the middle surface of the shell, choose an orthogonal curvilinear coordinate system $\left(\mathrm{x}_{1}, \mathrm{x}_{2}\right)$; the third coordinate is reported in the direction of the outer normal to the middle surface of the shell.

We will proceed from the variational equation [1]:

$$
\begin{aligned}
& \iint \dot{\Omega}\left(\mathrm{T}_{1} \delta \varepsilon_{1}+\mathrm{T}_{2} \delta \varepsilon_{12}+S_{12} \delta \varepsilon_{12} G_{1} \delta \mathrm{N}_{1}+G_{2} \delta \mathrm{N}_{2}+2 H_{12} \delta \mathrm{N}_{12}+Q_{1} \delta \varepsilon_{13} Q_{2} \delta \varepsilon_{23}\right) d \Omega \\
& +\int \Gamma\left[\mathrm{T}_{\rho}^{0} \delta u_{\rho}+s_{\rho 0}^{0} \rho u_{0}+G_{\rho}^{0} \delta \gamma_{a}+H_{\rho \theta}^{0} \delta_{\gamma \theta}+Q_{\rho}^{0} \delta \omega\right] d \Gamma=0,
\end{aligned}
$$

where $\Gamma=\Gamma_{1} \Gamma_{2} ; \Gamma_{1}, \Gamma_{2}$-outlines of holes; $\Omega$ - area, the boundary of which is far enough from the contours of the holes, and on it the damping conditions are satisfied; $\mathrm{T}_{1 \ldots}, Q_{2}$ components of the disturbed stress state; $\mathrm{T}_{\rho}^{0}, \ldots Q_{\rho}^{0}$-components of the main stress state.

\section{Results and discussion}

Table 1 shows the results of the study of the concentration coefficients of the annular forces $k_{\theta}^{T}$ (upper row) and $k_{\theta}^{M}$ (lower row) at various points of the hole contour, depending on the distance between the holes $/ / \sqrt{R h}$. The shell parameters were chosen as follows:

$\frac{r_{01}}{R}=0.1, \frac{h}{r_{01}}=0.1, \frac{r_{02}}{R}=0.075, \vartheta_{1}=0.3, \frac{E_{1}}{E_{2}}=0.8, \frac{G_{13}}{E_{1}}=\frac{G_{23}}{E_{1}}=0.1 \frac{G_{12}}{E_{1}}=0.23$ 
As can be seen from the table, the stresses on the contour of the larger hole at the point $\theta=0$ up to the distance $\sqrt[l]{R h}=1.5$ hardly change. With further approach of the holes to $l / \sqrt{R h}=0.25$ and the coefficient $k_{\theta}^{M}$ at the points $\theta=0, \pi / 2$ decreases, and at the point $\theta=\pi$, it increases. Of interest is the influence of the radius of the larger holes and the value of the jumper $\mathrm{d}$ on the stresses at the point $\theta=0$ of the contour of the small holes. This can be seen from the first column of the table. When the holes approach each other at the point $\theta=\pi$ on the contour of the larger hole, the stress concentration $k_{\theta}^{T}$ and $k_{\theta}^{M}$ increase by $131 \%$ and $102 \%$, respectively. Note that when the holes approach each other in the considered example, a non-monotonic character of the change in the stress concentration coefficients on the hole contours is also observed. A similar phenomenon was previously found for an isotropic shell with equal holes [7, 8].

Table 1. Concentration coefficients of the annular forces at various points of the hole contour.

\begin{tabular}{|c|c|c|c|c|c|c|c|c|c|}
\hline \multirow{2}{*}{$\theta$} & \multicolumn{5}{|c|}{$l / \sqrt{R h}}$, & \multicolumn{4}{|c|}{$r_{02} / \sqrt{R h}=0.75$} \\
\hline & 0.25 & 0.50 & 0.75 & 1.0 & 1.5 & 2.0 & 2.5 & 3.0 & 3.5 \\
\hline \multirow[t]{2}{*}{0} & 5.033 & 4.930 & 4.905 & 4.905 & 4.932 & 4.959 & 4.974 & 4.978 & 4.985 \\
\hline & 1.670 & 1.790 & 1.822 & 1.826 & 1.803 & 1.777 & 1.761 & 1.755 & 1.746 \\
\hline \multirow{2}{*}{$\frac{\pi}{4}$} & 3.319 & 3.264 & 3.250 & 3.247 & 3.255 & 3.267 & 3.273 & 3.276 & 3.278 \\
\hline & 0.727 & 0.786 & 0.805 & 0.812 & 0.809 & 0.802 & 0.796 & 0.793 & 0.791 \\
\hline \multirow{2}{*}{$\frac{\pi}{2}$} & 4.578 & 4.560 & 4.548 & 4.540 & 4.525 & 4.515 & 4.507 & 4.499 & 4.498 \\
\hline & 1.286 & 1.330 & 1.372 & 1.408 & 1.467 & 1.500 & 1.512 & 1.512 & 1.512 \\
\hline \multirow{2}{*}{$\frac{3 \pi}{4}$} & 2.276 & 2.291 & 2.852 & 2.927 & 3.068 & 3.178 & 3.264 & 3.339 & 3.340 \\
\hline & 0.407 & 0.466 & 0.525 & 0.579 & 0.654 & 0.670 & 0.641 & 0.592 & 0.591 \\
\hline \multirow[t]{2}{*}{$\pi$} & 9.447 & 6.313 & 5.374 & 4.943 & 4.720 & 4.819 & 4.953 & 5.050 & 5.050 \\
\hline & 3.190 & 2.260 & 1.932 & 1.774 & 1.664 & 1.658 & 1.646 & 1.615 & 1.601 \\
\hline
\end{tabular}

In Fig. 2 and 3 show the results of the study of stress concentration $k_{\theta}^{T}$ and $k_{\theta}^{M}$ at different values of the shear parameter $G_{23} / G_{13}$.. The shell parameters were chosen as in the case of Table 1 . Curves 1,2 , and 3 respectively refer to the points $\theta=0, \pi / 2, \pi$. It can be seen that the influence of $G_{23} / G_{13}$ on the stress concentration is most pronounced at the points $\theta=0$ and $\theta=\pi$.

The coefficients $k_{\theta}^{T}$ and $k_{\theta}^{M}$ with a change in the parameter ${ }^{23} / G_{13}$, from 0 to 0.1 at the point $\theta=0$ on the contour of the larger hole $\left(r_{01}\right)$ they increase by $10 \%$, and at the point $\theta=\pi$ by $7 \%$. At the point $\theta=\pi / 2$ on both contours of the holes, the influence of $G_{23} / G_{13}$

$G_{13}$ is insignificant. On the contour of the small hole at the points $\theta=0$ and $\theta=\pi$ with

a change in ${ }^{G_{23}} / G_{13}$, the coefficient $k_{\theta}^{T}$ increases by $11 \%$ and $5 \%$, respectively. Solid curves refer to hole $r_{01}$, and dashed curves to hole $r_{02}$. 


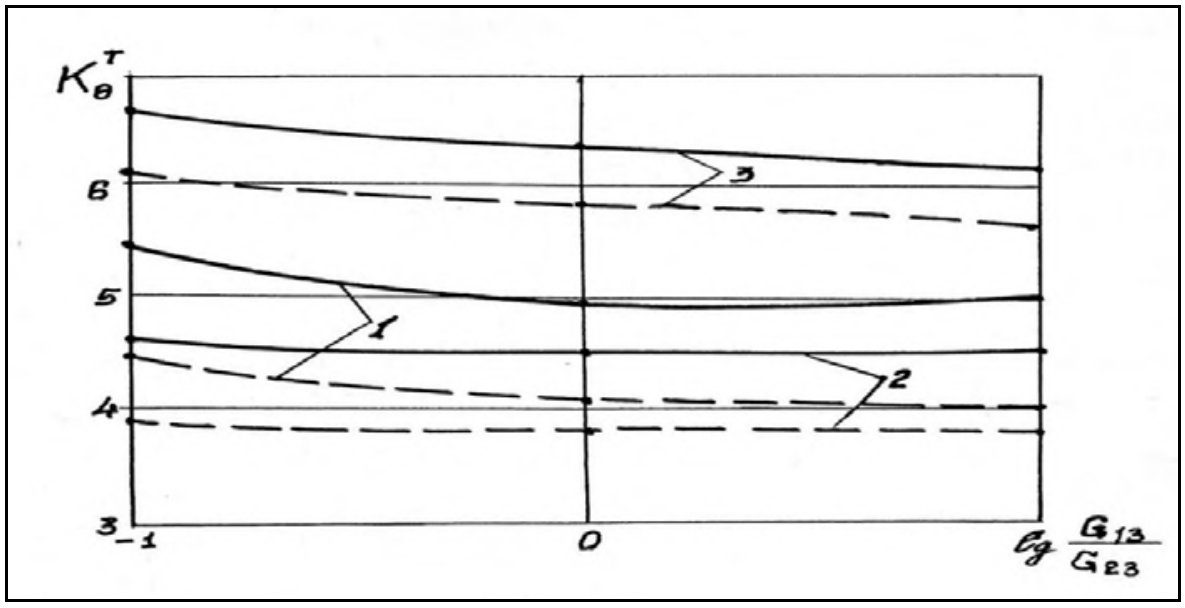

Fig. 2. Stress concentration $k_{\theta}^{T}$.

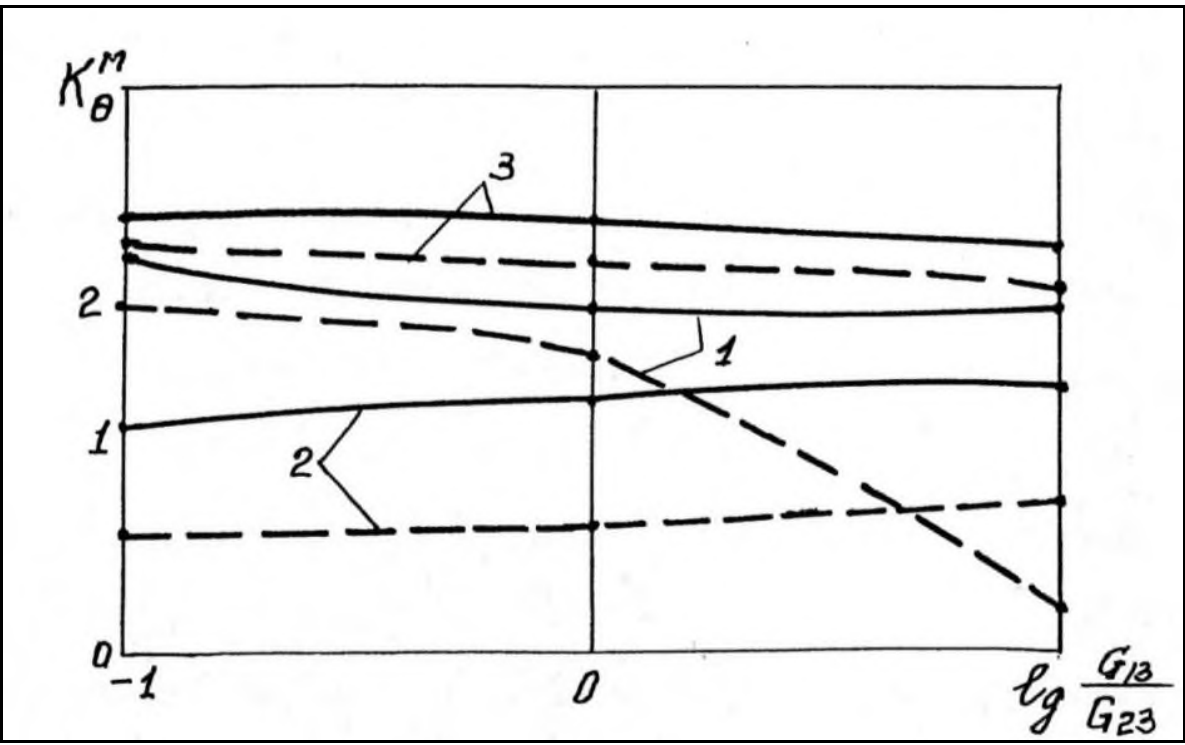

Fig. 3. Stress concentration $k_{\theta}^{M}$.

The effect of orthotropy of the material on the stress concentration $k_{\theta}^{T}$ (top line) and $k_{\theta}^{M}$ (bottom line) at various points of the hole contour is given in Table 2. The radius of one hole was fixed $r_{01} /{ }_{R}=0.1$, and the second hole $r_{02} /{ }_{R}=0.075$. It is seen that the orthotropy of the material significantly affects the stress concentration along both contours $E_{1} / E_{2}$ of the holes. With a change in the orthotropy of the material $E_{2}$ from 2.5 to 0.8 in and $25 \%$, respectively, and $k_{\theta}^{M}$ by $61 \%$ and $40 \%$. 
Table 2. Stress concentration $k_{\theta}^{T}$ (top line) and $k_{\theta}^{M}$ (bottom line) at various points of the hole contour.

\begin{tabular}{|c|c|c|c|c|c|c|c|c|c|}
\hline \multirow[t]{2}{*}{$\theta$} & \multicolumn{3}{|c|}{$l / \sqrt{R h}}$, & \multicolumn{6}{|c|}{$r_{01} / \sqrt{R h}=1.0$} \\
\hline & 0.25 & $\mathbf{0 . 5 0}$ & 0.75 & 1.0 & 1.5 & 2.0 & 2.5 & 3.0 & 3.5 \\
\hline \multirow{2}{*}{0} & 4.384 & 4.092 & 4.019 & 4.024 & 4.108 & 4.186 & 4.204 & 4.236 & 4.245 \\
\hline & 1.406 & 1.653 & 1.723 & 1.728 & 1.676 & 1.620 & 1.601 & 1.577 & 1.565 \\
\hline \multirow{2}{*}{$\frac{\pi}{4}$} & 2.902 & 2.762 & 2.720 & 2.713 & 2.737 & 2.766 & 2.783 & 2.790 & 2.801 \\
\hline & 0.596 & 0.778 & 0.773 & 0.794 & 0.796 & 0.783 & 0.771 & 0.766 & 0.761 \\
\hline \multirow{2}{*}{$\frac{\pi}{2}$} & 3.808 & 3.857 & 3.881 & 3.888 & 3.887 & 3.860 & 3.845 & 3.828 & 3.819 \\
\hline & 0.695 & 0.804 & 0.931 & 1.054 & 1.239 & 1.332 & 1.350 & 1.354 & 1.352 \\
\hline \multirow{2}{*}{$\frac{3 \pi}{4}$} & 1.970 & 2.079 & 2.183 & 2.292 & 2.478 & 2.613 & 2.714 & 2.796 & 2.800 \\
\hline & 0.02 & 0.03 & 0.170 & 0.318 & 0.550 & 0.661 & 0.686 & 0.668 & 0.664 \\
\hline \multirow{2}{*}{$\pi$} & 9.835 & 5.885 & 4.550 & 4.003 & 3.805 & 3.991 & 4.189 & 4.312 & 4.320 \\
\hline & 3.066 & 1.988 & 1.607 & 1.462 & 1.431 & 1.484 & 1.511 & 1.502 & 1.500 \\
\hline
\end{tabular}

This increase on the contour of the small hole at the points $\theta=0$ and $\theta=\pi$ for the coefficient $k_{\theta}^{T}$ is $35 \%$ and $15 \%$, and for $k_{\theta}^{M} 140 \%$ and $32 \%$. At the point $\theta=\pi / 2$ with a change in $E_{1} / E_{2}$, the coefficients $k_{\theta \text { and }}^{T} k_{\theta}^{M}$ decrease.

Table 3 shows the results of studying the stress concentration factors $k_{\theta}^{T}$ and $k_{\theta}^{M}$ depending on the change in the radii of the holes $r_{02} / r_{01}$. In this case, the radius of one hole was fixed $r_{01} / R=0.1$, and the radius of the other hole was changed $r_{02} / r_{01}=0.5,1 \cdot 0,1 \cdot 5,2 \cdot 0,2.5$. As the radius of the second hole increases, the stress concentration increases on the contour of both holes. When the parameter $r_{02} / r_{01}=0.5,1 \cdot 0,1.5,2 \cdot 0,2.5$ changes at the points $\theta=\pi / 2$ and $\theta=\pi$ on the contour of the first hole $\left(r_{01}\right)$, the coefficient $k_{\theta}^{T}$ increases by $6 \%$ and $21 \%$, respectively, and at the point $\theta=0$ decreases by $10 \%$. 
Table 3. Stress concentration factors $k_{\theta}^{T}$ and $k_{\theta}^{M}$ depending on the change in the radii of the holes.

\begin{tabular}{|c|c|c|c|c|c|c|c|c|}
\hline \multirow{3}{*}{$\theta$} & \multicolumn{8}{|c|}{$E_{1 / E_{2}}$} \\
\hline & \multicolumn{4}{|c|}{$r_{01} / R=0.125$} & \multicolumn{4}{|c|}{$r_{02} / R=0.10$} \\
\hline & 0.8 & 1.0 & 1.3 & 2.5 & 0.8 & 1.0 & 1.3 & 2.5 \\
\hline \multirow[t]{2}{*}{0} & 4.930 & 4.556 & 4.118 & 3.441 & 4.092 & 3.806 & 3.479 & 2.973 \\
\hline & 1.390 & 1.559 & 1.303 & 0.836 & 1.653 & 1.414 & 1.154 & 0.695 \\
\hline \multirow{2}{*}{$\frac{\pi}{6}$} & 3.634 & 3.666 & 3.681 & 3.709 & 3.084 & 3.107 & 3.175 & 3.242 \\
\hline & 1.061 & 1.032 & 0.998 & 0.890 & 0.897 & 0.935 & 0.870 & 0.710 \\
\hline \multirow{2}{*}{$\frac{\pi}{4}$} & 3.264 & 3.412 & 3.531 & 3.755 & 2.762 & 2.896 & 3.010 & 3.263 \\
\hline & 0.786 & 0.821 & 0.881 & 0.983 & 0.723 & 0.741 & 0.754 & 0.743 \\
\hline \multirow{2}{*}{$\frac{\pi}{3}$} & 3.444 & 3.601 & 3.776 & 4.176 & 2.924 & 3.086 & 3.246 & 3.637 \\
\hline & 0.926 & 0.977 & 1.049 & 1.210 & 0.778 & 0.805 & 0.824 & 0.850 \\
\hline \multirow{2}{*}{$\frac{\pi}{2}$} & 4.560 & 4.661 & 4.760 & 4.934 & 3.857 & 3.947 & 4.019 & 4.153 \\
\hline & 1.330 & 1.351 & 1.397 & 1.524 & 0.804 & 0.774 & 0.749 & 0.700 \\
\hline \multirow{2}{*}{$\frac{2 \pi}{3}$} & 3.331 & 3.486 & 3.674 & 4.156 & 2.531 & 2.666 & 2.816 & 3.204 \\
\hline & 0.680 & 0.700 & 0.735 & 0.836 & 0.067 & 0.035 & 0.009 & 0.001 \\
\hline \multirow{2}{*}{$\frac{3 \pi}{4}$} & 2.791 & 2.951 & 3.167 & 3.672 & 2.079 & 2.227 & 2.442 & 2.942 \\
\hline & 0.466 & 0.487 & 0.510 & 0.547 & 0.035 & 0.028 & 0.029 & 0.032 \\
\hline \multirow{2}{*}{$\frac{5 \pi}{6}$} & 3.244 & 3.380 & 3.545 & 3.838 & 2.279 & 2.958 & 3.172 & 3.553 \\
\hline & 0.694 & 0.715 & 0.727 & 0687 & 0.393 & 0.425 & 0.458 & 0.486 \\
\hline \multirow{2}{*}{$\pi$} & 6.313 & 5.990 & 5.616 & 4.986 & 5.985 & 5.722 & 5.534 & 5.206 \\
\hline & 2.260 & 2.111 & 1.919 & 1.550 & 2.005 & 1.878 & 1.733 & 1.444 \\
\hline
\end{tabular}

The coefficient $k_{\theta}^{M}$ on the contour of the first hole $r_{01}$ at the points $\theta=\pi / 2$ and $\theta=\pi$ decreases, and at the point $\theta=0$ it increases. At the points $\theta=0, \pi / 2, \pi$ on the contour of the second hole $r_{02}$ with an increase in the radius of the holes, the coefficient $k_{\theta}^{T}$ increases by $200 \%, 170 \%, 105 \%$, respectively, and the coefficient $k_{\theta}^{M}$ by $60 \%, 300 \%$, and $100 \%$.

Fig. 4-5 shows the distribution of stress concentration $k_{\theta}^{T}$ and $k_{\theta}^{M}$ at the points of the contour of the holes, depending on the effect of shear stiffness $G_{13} / E_{1}$. In this case, the radius of one hole was chosen $r_{01} /{ }_{R}=0.10$, and the other $r_{02} / R=0.15$. 


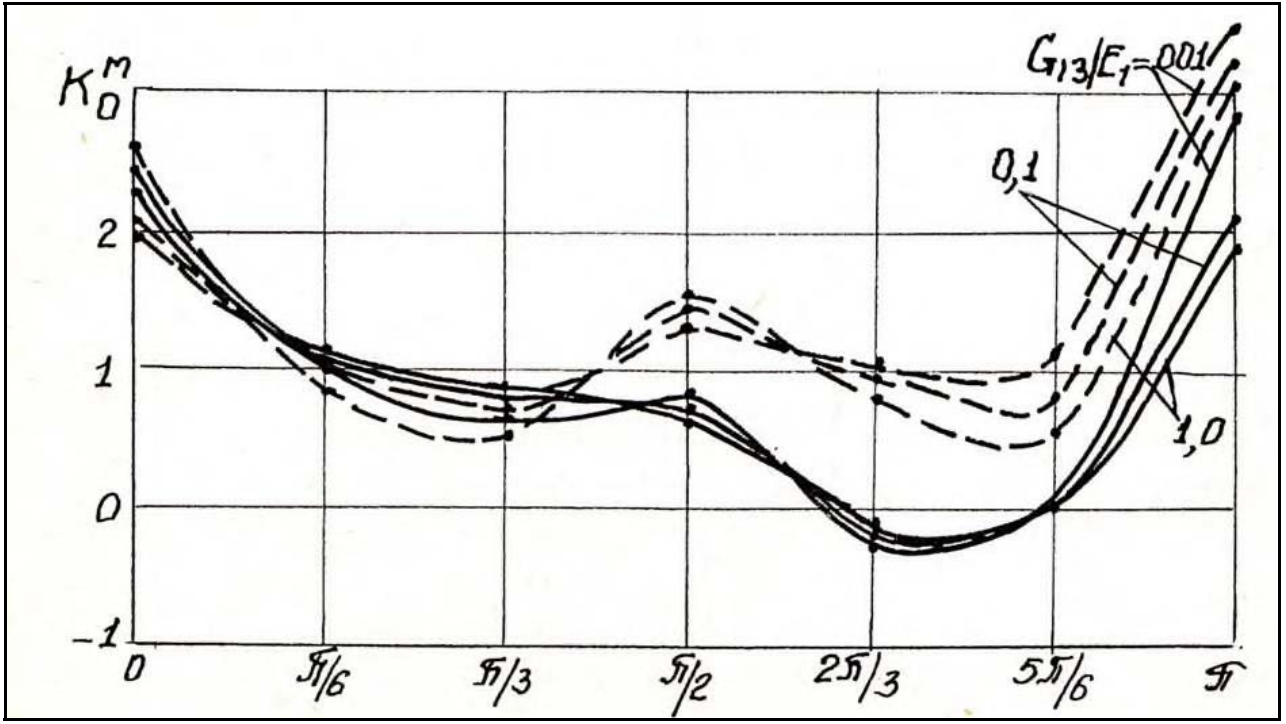

Fig. 4. Stress concentration $k_{\theta}^{T}$ at the points of the contour of the holes.

With a change in the shear stiffness $G_{13} / E_{1}$ from 1.0 to 0.01 at the points $\theta=0, \pi / 2$, $\pi$ on the contour $r_{01}$, the coefficient $k_{\theta}^{T}$ increases by $7 \%, 40 \%, 3 \%$, respectively, and the coefficient $k_{\theta}^{M}$ - by $8 \%, 4 \%, 30 \%$. At points $\theta=0, \pi / 2, \pi$ on the contour $r_{02}$ with a change in ${ }^{G_{13}} / E_{1}$, the coefficient $k_{\theta}^{T}$ increases, respectively, by $6 \%, 10 \%, 4 \%$, and the coefficient $k_{\theta}^{M}$ by $16 \%, 31 \%, 10 \%$.

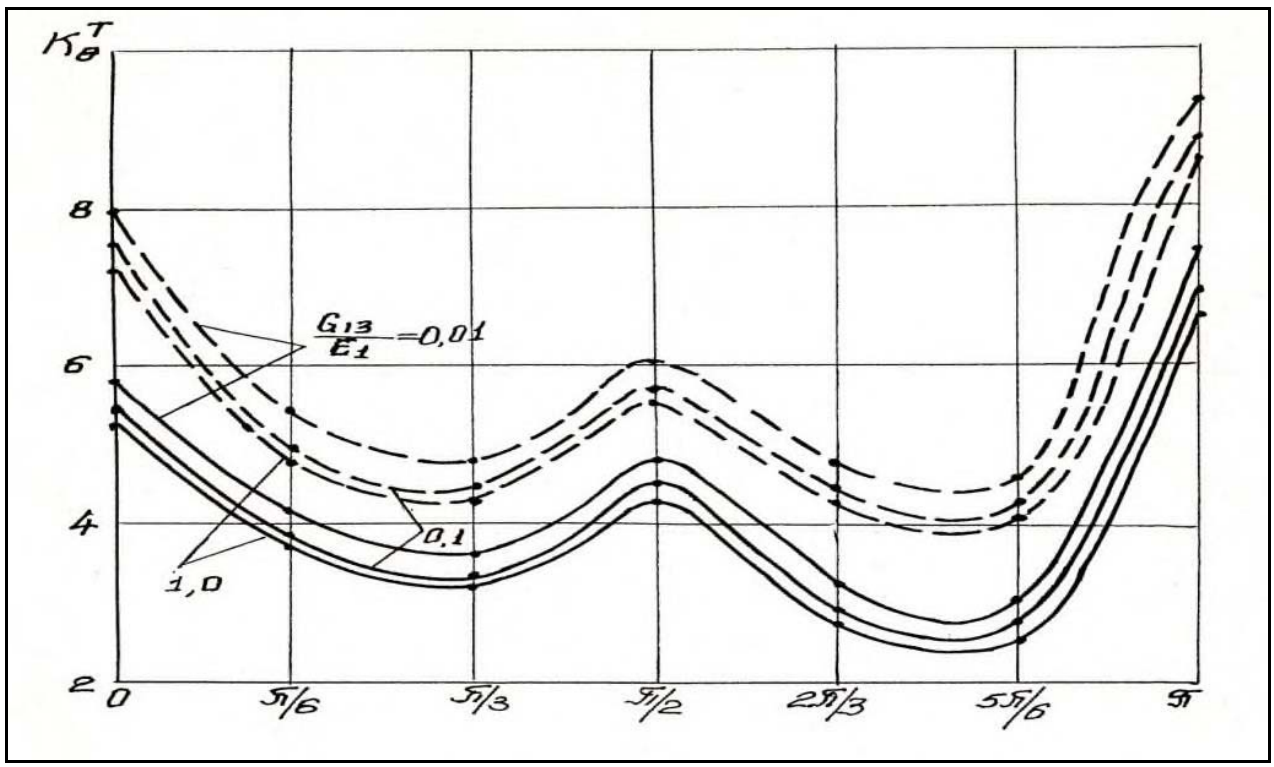

Fig. 5. Stress concentration $k_{\theta}^{M}$ at the points of the contour of the holes. 
Table 4 shows the results of a study of the concentration of annular forces $K_{\theta}^{T}$ and annular moments $K_{\theta}^{M}$ depending on the shell thickness $h / R$. The shell parameters were chosen as in the case of Table 1 . It can be seen that with decreasing shell thickness, the coefficients $k_{\theta}^{T}$ and $k_{\theta}^{M}$ on the contours of the holes $r_{01}$ and $r_{02}$ increase. At the points $\theta=0, \pi / 2, \pi$ on the contour of the larger hole $r_{01}$ with a decrease in thickness from 0.003 to 0.01 , the coefficient $k_{\theta}^{T}$ increases by $27 \%, 31 \%, 11 \%$, respectively, and the coefficient $k_{\theta}^{M}$ - by $32 \%, 24 \%, 3 \%$. By decreasing the shell thickness at the indicated points of the contour of the small hole $r_{02}$, the coefficient $k_{\theta}^{T}$ increases by $10 \%, 32 \%, 7 \%$, and the coefficient $k_{\theta}^{M}$ - by $50 \%, 42 \%, 7 \%$.

Table 4. Concentration of annular forces $k_{\theta}^{T}$ and annular moments $k_{\theta}^{M}$.

\begin{tabular}{|c|c|c|c|c|c|c|}
\hline \multirow{3}{*}{$\theta$} & \multicolumn{6}{|c|}{$h / R$} \\
\hline & \multicolumn{3}{|c|}{$r_{01} / R_{R}=0.125$} & \multicolumn{3}{|c|}{$r_{02} / R_{R}=0.10$} \\
\hline & 0.01 & 0.02 & 0.03 & 0.01 & 0.02 & 0.03 \\
\hline \multirow{2}{*}{0} & 4.930 & 4.153 & 3841 & 4.092 & 3.767 & 3690 \\
\hline & 1.790 & 1.519 & 1.367 & 1.653 & 1.102 & 1.081 \\
\hline \multirow{2}{*}{$\frac{\pi}{6}$} & 3.634 & 3.059 & 2.818 & 3.084 & 2.827 & 2.738 \\
\hline & 1.061 & 0.920 & 0.835 & 0.987 & 0.765 & 0.667 \\
\hline \multirow{2}{*}{$\frac{\pi}{4}$} & 3.264 & 2.717 & 2.491 & 2.762 & 2.472 & 2.355 \\
\hline & 0.786 & 0.694 & 0.636 & 0.723 & 0.532 & 0.463 \\
\hline \multirow{2}{*}{$\frac{4}{\frac{\pi}{3}}$} & 3.444 & 2.861 & 2.617 & 2.924 & 2.579 & 2.424 \\
\hline & 0.926 & 0.783 & 0.705 & 0.778 & 0.570 & 0.500 \\
\hline \multirow{2}{*}{$\frac{\pi}{2}$} & 4.560 & 3.792 & 3.424 & 3.857 & 3.198 & 2.885 \\
\hline & 1.330 & 1.162 & 1.067 & 0.804 & 0.623 & 0.561 \\
\hline \multirow{2}{*}{$\frac{2 \pi}{3}$} & 3.331 & 2.676 & 2.378 & 2.531 & 2.003 & 1.770 \\
\hline & 0.680 & 0.650 & 0.624 & 0.067 & 0.138 & 0.173 \\
\hline \multirow{2}{*}{$\frac{3 \pi}{4}$} & 2.791 & 2.286 & 2.071 & 2.079 & 1.788 & 1.664 \\
\hline & 0.466 & 0.498 & 0.503 & 0.035 & 0.154 & 0.210 \\
\hline \multirow{2}{*}{$\frac{5 \pi}{6}$} & 3.244 & 2.861 & 2.676 & 2.279 & 2.702 & 2.620 \\
\hline & 0.694 & 0.747 & 0.773 & 0.393 & 0.576 & 0.655 \\
\hline \multirow[t]{2}{*}{$\pi$} & 6.313 & 5.874 & 5.659 & 5.985 & 5.698 & 5.608 \\
\hline & 2.260 & 2.239 & 2.190 & 2.075 & 2.160 & 2.010 \\
\hline
\end{tabular}

For the same shell, Table 5 shows the nature of changes in the coefficients $k_{\theta}^{T}$ and $k_{\theta}^{M}$ from the shell radius. It is seen that with an increase in the shell radius $R / r_{01}$, the stress concentration factor decreases along both contours of the holes. With an increase in the shell radius $R / r_{01}$ from 5 to 20 at the points $\theta=0, \pi / 2, \pi$ on the contour of the larger hole $r_{01}$, the coefficient $k_{\theta}^{T}$ decreases by $16 \%, 36 \%, 84 \%$, respectively, and the coefficient $k_{\theta}^{M}$ - by $27 \%, 26 \%, 14 \%$. With an increase in the radius of the shell at the indicated points on the contour of a small hole $r_{02}$, the coefficient $k_{\theta}^{T}$ decreases by $26 \%, 36 \%, 10 \%$, and the coefficient $k_{\theta}^{M}$ decreases by $47 \%, 43 \%, 7 \%$. 
Table 5. Nature of changes in the coefficients $k_{\theta}^{T}$ and $k_{\theta}^{M}$.

\begin{tabular}{|c|c|c|c|c|c|c|c|c|}
\hline \multirow{3}{*}{$\theta$} & \multicolumn{8}{|c|}{$R / r_{01}$} \\
\hline & \multicolumn{4}{|c|}{$r_{01} / R^{=0.1}$} & \multicolumn{4}{|c|}{$r_{02} /_{R}=0.075$} \\
\hline & 0.2 & 0.1 & 0.06 & 0.05 & 0.2 & 0.1 & 0.06 & 0.05 \\
\hline \multirow{2}{*}{0} & 6.290 & 4.930 & 4.419 & 4.125 & 5.034 & 4.092 & 3.852 & 3.749 \\
\hline & 1.950 & 1.790 & 1.595 & 1.446 & 1.996 & 1.653 & 1.360 & 1.064 \\
\hline$\pi$ & 4.836 & 3.634 & 3.262 & 3.029 & 3.915 & 3.084 & 2.908 & 2.805 \\
\hline 6 & 0.996 & 1.061 & 0.991 & 0.939 & 1.061 & 0.987 & 0.856 & 0.772 \\
\hline$\frac{\pi}{-}$ & 4.416 & 3.264 & 2.901 & 2.683 & 3.558 & 2.762 & 2.565 & 2.445 \\
\hline$\overline{4}$ & 0.636 & 0.786 & 0.754 & 0.728 & 0.766 & 0.723 & 0.624 & 0.557 \\
\hline$\frac{\pi}{-\pi}$ & 4.551 & 3.444 & 3.051 & 2.822 & 3.721 & 2.924 & 2.693 & 2.545 \\
\hline$\overline{3}$ & 0.926 & 0.926 & 0.867 & 0.819 & 0.941 & 0.778 & 0.665 & 0.587 \\
\hline$\frac{\pi}{2}$ & 5.792 & 4.560 & 4.048 & 3.730 & 4.918 & 3.857 & 3.421 & 4.142 \\
\hline$\overline{2}$ & 1.493 & 1.331 & 2.202 & 1.123 & 1.065 & 0.804 & 0.667 & 0.616 \\
\hline $2 \pi$ & 4.498 & 3.331 & 2.887 & 2.618 & 3.530 & 2.531 & 2.135 & 1.958 \\
\hline$\overline{3}$ & 0.739 & 0.680 & 0.665 & 0.664 & 0.060 & 0.067 & 0.127 & 0.182 \\
\hline $3 \pi$ & 3.783 & 2.791 & 2.438 & 2.240 & 2.671 & 2.079 & 1.8712 & 1.758 \\
\hline$\overline{4}$ & 0.428 & 0.466 & 0.500 & 0.527 & 0.092 & 0.035 & 0.132 & 0.201 \\
\hline $5 \pi$ & 4.044 & 3.244 & 2.976 & 2.817 & 2.997 & 2.279 & 2.728 & 2.673 \\
\hline$\overline{6}$ & 0.579 & 0.694 & 0.747 & 0.781 & 0.198 & 0.393 & 0.5666 & 0.576 \\
\hline \multirow[t]{2}{*}{$\pi$} & 6.894 & 6.313 & 6.034 & 5.811 & 6.390 & 5.985 & 5.861 & 5.768 \\
\hline & 2.479 & 2.260 & 2.181 & 2.144 & 2.136 & 2.005 & 1.990 & 2.001 \\
\hline
\end{tabular}

\section{Conclusions}

In this paper, we studied the stress distribution around two unequal circular holes in an orthotropic spherical shell made of a composite material. Using the refined theory of Timoshenko-type shells, we considered the effect of transverse shear deformations for the entire package of the shell as a whole. In this case, the composite material is considered as homogeneous with the given characteristics.

Thus, the developed technique can be used to calculate the elements of shell structures made of composite materials with several holes.

\section{References}

1. A. N. Guz, I. S. Chernyshenko, V. M. Chekhov, K. I. Sherenko, Methods for Calculating Shells, 8(1), 636 (2000)

2. A. Guz, K. Shnerenko, M. Ryndyuk, Scientific Comprehension, 48 (1992) (in Russian)

3. R. Gallager, Finite Element Method, 4(14), 48-62 (2014)

4. A. Guz, I. Chernyshenko, K. Shnerenko, Scientific Comprehension, 323 (1990) (in Russian)

5. N. Noraliev, Stress State of an Orthotropic Spherical Shell, Weakened by Two Circular Holes, 8, 47-50 (1990)

6. K. Shnerenko, N. Noraliev, Stress Concentrations Around Two Unequal Holes in an Orthotropic Spherical Shell Made of Composite Material, 5, 20-22 (1999)

7. K. Shnerenko, N. Noraliev, Sci. Conf. Young Scientists, 5166, 451-455 (1989) (in Russian) 
8. N. Noraliev, N. Indiaminov, Applied Mechanics, 26(12), 105-108 (1990)

9. K. Shnerenko, V. Godzula, Mechanics of Composite Materials, 39(4), 651-652 (2013)

10. K. Shnerenko, Applied Mechanics, 42(5), 73-75 (2016)

11. A. Abdurashidov, K. Shnerenko, N. Noraliev, Strength Problems, 9, 34-38 (1992) (in Russian)

12. K. Shnerenko, N. Noraliev, Application Modeling, 2, 23-27 (1991)

13. N. Noraliev, F. Kudaeva, Journal of Problems of Computational and Applied Mathematics, 4, 61-65 (2018)

14. B. Rakhmankulova, K. Djamalov, S. Ziyaeva, IOP Conference Series: Materials Science and Engineering, 614(2), 012207 (2020) 